A strategically timed verbal task improves performance and neurophysiological alertness during fatiguing drives

\author{
Paul Atchley ${ }^{1}$, Mark Chan ${ }^{2}$, and Sabrina Gregersen ${ }^{1}$, \\ ${ }^{1}$ Department of Psychology \\ University of Kansas \\ ${ }^{2}$ Department of Psychology \\ Shenandoah University
}

\title{
Précis
}

Engagement in a concurrent task, such as a verbal task while driving has been found to improve driving performance during periods of monotonous driving. This study extends this research to longer, fatiguing monotonous drives. Neurophysiological recordings show that a verbal task improved driver alertness when performance also improved.

Word count (excluding, tables, figures, and references): 4458

\section{Editorial Correspondence}

Paul Atchley Department of Psychology 1415 Jayhawk Boulevard Lawrence, KS 66045

USA patchley@ku.edu 


\section{ACKNOWLEDGEMENTS}

This study was partially funded by a grant from the Kansas University Transportation Research Institute to the first author. Portions of this work were part of a senior thesis for the third author and were presented at the Cognitive Neuroscience Society 2010 annual meeting. 


\begin{abstract}
Objective: The objective of this study was to investigate if a verbal task can improve alertness and if performance changes are associated with changes in alertness as measured by EEG.

Background: Previous research showed that a secondary task can improve performance on a short, monotonous drive. The current work extends this by examining longer, fatiguing drives. The study also uses EEG to confirm that improved driving performance is concurrent with improved driver alertness. Method: A ninety minute, monotonous simulator drive was used to place drivers in a fatigued state. Four secondary tasks were used: No Verbal Task, Continuous Verbal Task, Late Verbal Task and a passive Radio Task. Results: When engaged in a secondary verbal task at the end of the drive, drivers showed improved lane keeping performance and had improvements in neurophysiological measures of alertness. Conclusion: A strategically timed concurrent task can improve performance even for fatiguing drives. Application: Secondarytask countermeasures may prove useful for enhancing driving performance across a range of driving conditions.
\end{abstract}

Key Words: countermeasures, monotony, fatigued driving, alertness, concurrent task, attention 


\section{Introduction}

Fatigue in a driving context manifests itself as drowsiness, a disinclination to keep driving, or in some cases falling asleep behind the wheel (Brown, 1994). The National Highway Transportation Safety Administration (NHTSA; 2011) reported an annual average of 83,000 fatigue related crashes per year from 2005 to 2009. Fatigue can be divided into two components: physiological fatigue and psychological fatigue. The former results from circadian influences or sleep deprivation, and the latter can occur under monotonous driving conditions even when drivers are rested (Thiffault \& Bergeron, 2003). Moreover, psychological fatigue often precedes physiological fatigue, resulting in performance decrements due to decreased alertness (Warm, Parasuraman, \& Matthews, 2008). Psychological fatigue is insidious as drivers are aware of their psychological state (Nordbakke \& Sagberg, 2007) yet still continue with their journey. Drivers often employ self-initiated passive countermeasures such as playing music or winding down the windows in an attempt to decrease fatigue (Gershon, Shinar, Oron-Gilad, Parmet, \& Ronen, 2011). However, Horne and Reyner (1998) report that the effects of these methods are often short-lived. Stopping to take a break or a nap is the best option to decrease the effects of fatigue (Driskell \& Mullen, 2005; Nguyen, Jauregui, \& Dinges, 1998).

A number of countermeasures to combat fatigue and loss of alertness have been examined. Alertness is defined here as driver awareness of their performance, such as lane keeping and awareness of their immediate environment, such as signs and other objects. Examples of countermeasures include visual search and auditory memory tasks (Engström, Johansson, \& Östlund, 2005), mental orienting puzzles (Liang \& Lee, 2009), foreign language learning (Takayama \& Nass, 2008), or games (Oron-Gilad, Ronen, \& Shinar, 2008; Verwey, \& Zaidel, 1999). These tasks are cognitively complex, requiring memory, spatial, or linguistic 
operations to perform. Though they have been shown to be effective at improving driver alertness, the question remains what the minimum conditions are for countermeasures to improve alertness. Even fairly simple tasks may improve driver alertness. For example, drivers making periodic verbal reports of odometer readings during a seven hour drive exhibited better performance than non-engaged drivers (Drory, 1985).

Drivers may feel a cell-phone conversation is a good countermeasure candidate. OronGilad \& Shinar (2000) report military truck drivers list engaging in a cell-phone conversation useful in countering the effects of fatigue. Gershon et al. (2011) noted that conversations with a passenger were a preferred method to maintain alertness among non-commercial drivers. Yet the driving safety literature reports an increase in crash risk because of distraction from using the cell phone. Decreased visual attention, poorer vehicular control, slower reaction times are among some of the decrements in driving performance when distracted (Atchley \& Dressel, 2004; Rakauskas, Gugerty, \& Ward, 2004; Strayer \& Drews, 2007) leading to a fourfold increase in crash risk while talking on the phone while driving, even hands-free (McEvoy et al., 2005; Redelmeier \& Tibshirani, 1997).

This study builds on previous work examining the use of low-demand, interactive, verbal tasks to improve driver alertness, to a longer drive where loss of alertness is more pronounced. A second goal is to confirm behavioral effects with physiological measures. In a previous study (Atchley\& Chan, 2011), we reported that introducing a verbal task at the end a short monotonous drive improved performance. Engaging in the verbal task the entire drive did not result in a performance sparing, suggesting the task must be strategically timed. As opposed to tasks described previously (Engström, Johansson, \& Östlund, 2005; Liang \& Lee, 2009; Oron-Gilad, Ronen, \& Shinar, 2008; Takayama \& Nass, 2008; Verwey, \& Zaidel, 1999), this task demanded 
very little working memory, suggesting that a task of minimal complexity can improve driver alertness. The drive was designed to induce monotony rather than fatigue the drivers, so it was relatively short in duration (around 25 minutes) (Desmond, Hancock, \& Monette, 1998; Lal \& Craig, 2002). It is unclear if the verbal countermeasure will work for longer, fatigue inducing, drives which are more typical for motor carrier operators, military operations, or long-distance recreational drives. Second, improved performance was measured primarily by lateral vehicle control. While maintaining a stable lane position is certainly important for safe driving, one must infer that lane keeping indicates increased alertness.

There are neurophysiological signatures of alertness that might provide more direct evidence that a verbal task improves driving by improving alertness. An increase in task demand can result in increased alertness as measured by psychophysiological measures such as EEG (Brookhuis \& De Waard, 1993; Oron-Gilad, Ronen, \& Shinar, 2008) and heart rate variability (Gershon, Ronen, Oron-Gilad, \& Shinar, 2009; Mehler, Reimer, Coughlin, \& Dusek, 2009). Brookhuis and De Waard (1993) investigated driver alertness levels by measuring changes in beta, alpha and theta activity. They found that alertness declined over the duration of a task and was accompanied by a decline in driver performance. Oron-Gilad, Ronen, \& Shinar (2008) found that introducing a trivia game improved performance and alertness as measured by EEG. Gershon, et al. (2009) showed that improved lane keeping was accompanied by reductions in heart-rate variability for the same type of task. In the current work, we will use a similar method to validate the earlier results.

We hypothesize that an interactive verbal task (a task that requires listening and responding verbally) will improve driving performance when it is strategically timed for the period of greatest fatigue, rather than performed continuously throughout the drive. Further, 
there will be a convergence of behavioral and electrophysiological (EEG) data suggesting the interactive secondary task improves driver alertness. These results would establish that an interactive verbal task can improve safety and that behavioral measures previously shown to be measures of driver performance are useful even when drivers are engaging in secondary tasks.

\section{METHOD}

\section{Participant (Drivers)}

Sixty-nine undergraduates from the university's research pool participated for course credit. Drivers were randomly assigned into one of four groups: No Verbal Task, Late Verbal Task, Continuous Verbal Task, and Radio Show, with ten drivers per group. The final dataset had 25 males and 15 females $(M=19.4$ years, $S D=1.17)$. Drivers had an average of 4.3 years of driving experience $(S D=1.41)$. The data for the remaining 29 participants were excluded due to excessive movement artifacts and equipment failure.

\section{Materials and apparatus}

Driving simulator. The driving scenario was designed using STISIM Drive (Systems Technology Inc. Hawthorne, CA) simulator software (Version 2.08.02). A fixed-base cockpit with force-feedback steering and pedals was used. The vehicle was set on automatic transmission. Drivers viewed the scenario on a single 20 inch LCD display, giving a $45^{\circ}$ field of view. A Fresnel lens was used to improve the sensation of depth.

Scenario. The roadway was four-lane rural highway separated by a median. Lane width was 3.66 meters and visibility was set at 457.2 meters under clear conditions. The roadway was generally flat and monotonous with minimal traffic. To allow overtaking, traffic in the driver's lane travelled slower than the driver. The roadway was designed to mimic under-stimulating 
driving conditions that most drivers encounter on rural highways (Thiffault \& Bergeron, 2003).

The road was straight and flat, with one curve per block. Drivers passed 4 cars on average per block. Total driving distance was $178.200 \mathrm{~km}$ which included a $0.600 \mathrm{~km}$ start-up distance to allow drivers to attain the minimum speed of $112.65 \mathrm{~km} / \mathrm{h}$. An auditory warning sounded if driving speed exceeded $125.53 \mathrm{~km} / \mathrm{h}$. Data collection began at the $0.600 \mathrm{~km}$ mark and ended at the $177.600 \mathrm{~km}$ mark. Data was divided into 9 blocks of $19.733 \mathrm{~km}$.

Braking events occurred in the first, fourth, seventh and ninth block of the drive to ensure attentiveness. A large stop sign would suddenly appear and occlude the driving scene requiring the driver to respond as quickly as possible by bringing the car to a complete stop. Billboards of popular North American fast food restaurants were presented on the right shoulder in each driving block. This served as a memory test and additional evidence of concurrent task costs during the drive (Strayer \& Drews, 2007). Drivers were not informed about the memory task.

Concurrent verbal task. The verbal task was designed to capture the listening and production aspects of a conversation with minimal memory load. This task was based on previous work (Atchley \& Dressel, 2004). Word-game type tasks may produce larger effects on concurrent attention tasks than actual conversations due to the inability of participants to deprioritize the task in the same way they can do with actual conversations. Kunar, Carter, Cohen, \& Horowitz (2008) found that a word-game (form a new word using the last letter of a target word) led to slower RTs on a multiple-object tracking task than a conversation (see Atchley, Jones, Burson, \& Marshall, 2011, for additional discussion). The current work simplified this task by using a word-association task. Seventy-one prerecorded English words with positive valence and low arousal, spoken by a male native-speaker of English, were chosen from the ANEW wordlist (Bradley \& Lang, 1999). The words (randomly selected without replacement 
until list completion) were presented to the right ear of the driver. Drivers were informed the task was to free associate a one-word response to the stimulus and that there were no incorrect responses. The software automatically continued with the next word after 4 seconds. Late Verbal Task drivers engaged in the verbal task only in the last block. Continuous Verbal Task drivers engaged in the verbal task continuously in the first block, and two minutes in subsequent blocks. The onset of the verbal task in the subsequent blocks was randomized. No Verbal Task drivers had no secondary task. Radio Show drivers listened to episodes of "This American Life" for the duration of the drive.

Electrophysiological recording. EEG activity was continuously sampled at $500 \mathrm{~Hz}$ using a BioPac MP150 amplifier from electrodes placed on T4 and P4 with the ground electrode placed on the right mastoid in accordance to the international 10-20 system (Jasper, 1958). A high band-pass filter was used to remove excessive noise and manual inspection of the data was used to remove blinks and other noise spikes.

\section{Procedure}

EEG recording electrodes were applied after consent procedures. Drivers completed a demographic datasheet and the Dundee Stress State Questionnaire (DSSQ) (Warm, Matthews, \& Finomore, 2008). Drivers turned off their cell phones and removed watches. After EEG baseline recording (a reference for manual artifact removal/participant removal due to excessive artifacts) in which participants rested in their seat with eyes open (one minute) and closed (one minute), drivers practiced driving for five minutes. The experimenter informed drivers to maintain their speed between $112.65 \mathrm{~km} / \mathrm{h}$ and $120.70 \mathrm{~km} / \mathrm{h}$ and to drive in the right lane unless overtaking another vehicle. Drivers were equipped with the hands-free device, practiced the verbal task for one minute and were informed that they might do a verbal task. The experimenter 
sat behind the driver for the duration of the drive to ensure active participation. There was no verbal interaction between driver and experimenter during the experiment. Upon completion of the drive, drivers completed the second half of the DSSQ and were given two minutes to freely recall the billboards that they saw while driving. The drive lasted approximately 90 minutes, and was conducted in a darkened room with no ambient light.

\section{Performance and alertness}

SDLP. Consistent with the previous study (Atchley\& Chan, 2011), we measured standard deviation of lane position (SDLP). Drivers exhibit poorer SDLP with increased time on task due to reduced alertness and attention to the driving task (Atchley \& Chan, 2011; Desmond \& Matthews, 1997). Greater SDLP values are also associated with increased collision risks (Ranney, Harbluk, \& Noy, 2005). As we will note in the discussion, there are alternative explanations for the interaction of SDLP and alertness.

Engagement index (E/I). Alertness was measured using a combination of EEG alpha, beta and theta power spectral bands. The formula alpha+theta/beta yields the "engagement index" (E/I). Decreases in E/I values suggest increased alertness (Berka, et al., 2007; Pope, Bogart, \& Bartolome, 1995; Prinzel, Freeman, Scerbo, Mikulka, \& Pope, 2000).

DSSQ. For the DSSQ, the factor of "Task engagement" was measured. The factors "Distress" and "Worry" were not investigated. Task engagement is influenced by energetic arousal and motivation (Matthews et al., 2010). Task engagement decreases as energy levels and motivation decline.

Attention and memory. Drivers encode less information from the environment as alertness declines. In the current study, drivers had to recall the billboards that they saw during the drive similar to the method of Strayer \& Drews (2007). 


\section{RESULTS}

The comparison of interest is how alertness changes before and after the introduction of the countermeasure task. Countermeasure drivers (late verbal task) were compared to drivers engaging in the task the entire drive, doing nothing, or engaging in a passive task. Late verbal task drivers and continuous verbal task drivers were performing the same task on the last block. Analyses were conducted between groups on a combination of the first and second time blocks to establish a baseline for each group. To investigate changes in alertness, analyses were conducted on the eighth and ninth time blocks (late) when the late verbal task was introduced both across groups on block nine and across time (between blocks eight and nine) for each group. Analysis were conducted using a 2 (Time) x 4 (Task) mixed factorial ANOVA unless otherwise stated. Holm-Bonferroni corrections were applied to multiple comparisons.

\section{SDLP}

Instances where drivers were overtaking another vehicle or where drivers drifted outside the lane were excluded from the analysis. Data for baseline SDLP are presented in Figure 1. There was a significant difference in lateral control between verbal task groups, $F(3,36)=2.95$, $p=.046, \eta_{p}^{2}=.197$. Post-hoc analysis (Tukey's HSD) indicated Continuous Verbal Task drivers exhibited lower lateral control $($ mean $=0.39$ meters $)$ than No Verbal Task drivers $($ mean $=0.46$ meters; $p=.031$ ). No other comparisons were significant (all $p$ 's $>.05$ ).

For performance across the last two blocks, there was a significant main effect of time, $F(1,36)=21.70, p<.001, \eta_{p}^{2}=.376$, and verbal task, $F(3,36)=3.83, p=.018, \eta_{p}^{2}=.242$.

There was a significant Time $\mathrm{x}$ Verbal Task interaction, $F(3,36)=47.13, p<.001, \eta^{2}=.797$. Paired sample t-tests between time block eight and nine revealed a significant decrease in lateral 
control behaviors only for Late Verbal Task (from a mean of 0.51 to 0.38 meters) drivers, $t(9)=$ $10.87, p<.001, d=2.60$. No other groups exhibited significant changes (all $p$ 's $>.05$ ).

There was no difference between verbal task groups at time block eight (all $p$ 's $>.05$ ). Analysis at time block nine indicated that Late Verbal Task drivers (mean $=0.38$ meters) were better than No Verbal Task (mean $=0.55$ meters), $t(18)=-6.39, p<.001, d=-3.091$, Continuous Verbal (mean $=0.49$ meters), $t(18)=-3.39, p=.016, d=-1.571$, and Radio Show $($ mean $=0.46$ meters $), t(18)=-2.80, p=.039, d=-1.33$, drivers. In addition, Radio Show drivers exhibited better lateral control than No Verbal Task drivers, $t(18)=-2.89, p=.039, d=$ 1.39. No other comparisons were significant (all $p$ 's $>.05$ ).

\section{Engagement index (E/I)}

Data for baseline engagement index are presented in Figure 2. Note that smaller values indicate improved alertness. The axis is reversed in Figure 2 so that increased alertness is shown by an increase in value in the figure. There was no main effect of verbal task, $F(3,36)=1.33, p$ $=.280, \eta_{p}^{2}=.100$, nor was there a interaction, $F(3,36)=1.26, p=.30, \eta_{p}^{2}=.095$.

For performance across the last two blocks, there was a significant main effect of Time, $F(1,36)=9.31, p=.004, \eta_{p}^{2}=.205$, and a significant Time $\mathrm{x}$ Verbal Task interaction, $F(3,36)=$ 7.02, $p<.001, \eta^{2}=.369$. There was no main effect of verbal task, $F(3,36)=1.17, p=.334, \eta_{p}^{2}$ $=.089$. The interaction was investigated testing the effect of time on each level of verbal task, and the effect of verbal load at each level of time. Paired sample t-tests found drivers in the Late Verbal Task group showed improved E/I values, $t(9)=4.23, p=.002, d=1.49$, between time blocks eight and nine (decreasing from 4.25 to 3.24 ). No other groups showed a significant change in E/I values (all $p$ 's $>.05$ ). 
Analysis at time block nine revealed better E/I values for drivers in the Late Verbal Task $($ mean $=3.24)$ than Radio Show drivers $($ mean $=4.59), t(18)=-3.14, p=.034, d=-1.44$. No other comparisons were significant (all $p$ 's $>.05)$.

\section{DSSQ}

The task engagement (TE) factor of the DSSQ was analyzed using a 2 (time: pre and post) x 4 (verbal task) mixed factorial ANOVA. There was a main effect of time, $F(1,36)=$ 216.93, $p<.001, \eta_{p}^{2}=.858$. There was a significant Time $\mathrm{x}$ Verbal Task interaction, $F(3,36)=$ $3.79, p=.018, \eta_{p}^{2}=.240$. There was no main effect of verbal task, $F(3,36)=0.45, p=.719, \eta_{p}^{2}$ $=.036$. The interaction was investigated testing the effect of time on each level of verbal task, and the effect of verbal load at each time block. Paired sample t-tests between pre and post task TE scores revealed a significant decline in task engagement for all task types (all $p$ 's $<.001$ ) (see Table 1). Analysis of task engagement scores pre and post task did not reveal significant difference between task types (all p's >.05).

\section{Billboard recall}

One-way ANOVA found a significant difference between tasks, $F(3,36)=5.94, p=$ $.002, \eta_{p}^{2}=.331$. Post-hoc analysis with Tukey’s HSD adjustments indicated that drivers in the Continuous Verbal task recalled fewer billboards $(\mathrm{M}=34.3 \%, \mathrm{SD}=22.5)$, than drivers in the No Verbal $(\mathrm{M}=64.3 \%, \mathrm{SD}=12.1, p=.005)$ and Late Verbal $(\mathrm{M}=64.3 \%, \mathrm{SD}=20.5, p=$ .005 ) task groups. Drivers in the Radio Show task did not differ from the other tasks (all $p$ 's $>$ .05) (see Table 2).

\section{Discussion}

The results replicate earlier work (Atchley \& Chan, 2011) and show that engaging in a verbal task does improve performance, even when a driver is fatigued following a long drive 
(around 80 to 90 minutes in the current study). All drivers reported feeling less engaged by the end of the drive. However, drivers in the late verbal task group exhibited improved lane keeping, compared to the other groups, including drivers who had been talking the entire trip. This boost in driving performance was mirrored by improved EEG signatures that indicate alertness. Taken together, the current data support the notion that an interactive verbal task improves driving performance by increasing driver alertness. The verbal task produced more gains than the passive secondary task. The data are consistent with previously cited work showing that secondary tasks can improve alertness and performance, and extend the finding to show that even relatively simple verbal exchanges that are low in memory load or context, can serve as countermeasures. Technological interventions to improve driver alertness need not be overly demanding of the driver, as long as they are interactive. Additional work to establish the absolute minimum conditions and sustainability of these effects is needed.

The data do not suggest, that conversing via a cellular phone is always beneficial. In the current study, one of the groups drove the entire trip while engaging in a verbal task. This group did experience relatively better lane keeping at the start of the study, but there was no concurrent difference in the engagement index for this group in the early blocks. Consistent with the recent work of Cooper, Medeiros-Ward and Strayer (in press), the verbal task may have moved attention away from steering processes and a tendency of drivers new to the simulator to overcorrect for novel steering dynamics. The distraction of the verbal task may have made drivers less aware of small changes in lane position, producing fewer overcorrections, and more stable driving performance. But drivers in the continuous verbal task group saw no improvement in performance or alertness in later blocks, even though they were performing the verbal task. Further, this group was distracted, as measured by their ability to attend to and recall roadside 
billboards. This group experienced a 30\% reduction in amount of information recalled following the drive. While failure to recall billboards does not represent a safety risk in itself, inattentiveness does lead to increased risk.

Two issues in the current work should be highlighted for further consideration. The first is the nature of the verbal task and what it means for development of countermeasures to promote alertness. The current task was designed to require the driver to listen and produce a verbal response using limited working memory resources. However, it was not a conversation. Unlike a conversation, the prompts continued even in the absence of driver responses. The results suggest that even a simple form of listening and responding can produce alertness gains. However, one issue that remains to be resolved are the precise parameters that produce this effect. In fact, one criticism of the field generally is that not enough has been done to understand the conversation-side of the issue, or standardize methodological practice regarding verbal secondary tasks. The task used here, with a limited set of prompts and a free-association response option, produces reliable effects. More work needs to be done to determine the underlying mechanisms by which verbal tasks produce effects.

The second issue is lateral control as a measure of alertness. Lateral control is commonly used as a measure of alertness in studies using driving simulation, particularly on straight road simulations (Oron-Gilad \& Ronen, 2007). As alertness declines, lateral control also declines. Here the decline was associated with declining alertness measured by EEG. Improved lateral control late in the drive for the late verbal task group was also associated with a change in EEG. However, the continuous verbal task group, also showed better lateral control at the start of the drive, compared to other groups. The finding that lateral control improves with the addition of a secondary task in the absence of monotony-related declines has been found elsewhere (see, for 
example, Engström, Johansson, \& Östlund, 2005; Liang \& Lee, 2010). This effect is not limited to driving simulation. Stoffregen, Pagulayan, Bardy, \& Hettinger (2000) report finding a similar effect of a secondary task on postural stability. Becic et al. (2010) suggest that the stability of lateral control may relfect the relatively insensitivity of the driver to updating visual information, rather than improved performance. Cooper, Medeiros-Ward and Strayer (in press) reach a similar conclusion and dissociate the effect from changes in gaze.

There are reasons to believe the current data do reflect changes in alertness. The first is the concomitant change in alertness as measured by EEG. The second is analysis of braking data for a subset of the current participants, presented in a previous work (Deboeck et al., 2011). Momentary derivative estimates of lateral velocity in addition to more traditional lateral control measures to estimate driver performance found a close correspondence between momentary estimates of lateral velocity and more gross changes in vehicle lateral control. Most importantly, both talking (late in the drive) and unexpected braking events during the drive produced reductions in small steering corrections that increase with drive time. In other words, the conversation produced the same effect on vehicle control as a sudden braking event, the latter which one would assume would alert a fatigued driver.

Given these data and the EEG data, improvement in SDLP at the end of the drive most likely reflects improved performance due to increased alertness. However, it is possible that early differences in SDLP for the continuous verbal task group reflect some other process. Shomstein, \& Yantis (2004) demonstrated decreased visual cortical activity during a conversation suggesting the verbal task might decrease sensitivity to the optic flow signals that would drive motion perception. This would also be with data showing restricted eye-movements with a concurrent task (Engström, Johansson, \& Östlund, 2005; Recarte, \& Nunes, 2000). Or, 
response output bottlenecks may limit the number of corrections a driver can engage in while simultaneously performing a verbal task. Clearly the interaction between commonly used measures of driver performance and secondary tasks needs to be examined more systematically. The current data indicate both benefits and costs for performing a secondary task while driving. The benefits of re-alerting of drivers with the secondary task and the costs occur when a secondary task loses its novelty due to over-performance and the task interferes with attention. To understand how secondary tasks aid performance, we need to understand how a secondary task interacts with sustained attention. It is less than clear how secondary tasks interact with declines in sustained attention over time because of multiple views of what causes such declines. One view of sustained attention suggests that performance decrements are due to the understimulating nature of the primary task (Cerezuela et al., 2004; Manly, et al., 1999; Robertson, et al., 1997; Wertheim, 1978). Reduced cerebral blood flow to cortical and subcortical regions with increasing time-on-task has been suggested to indicate that under-stimulation reduces resources necessary for peak performance (Foucher, Otzenberger, \& Gounot, 2004; Paus, et al., 1997). Additional stimulation may increase the metabolic resources needed, consistent with the data of the late verbal task group in the current study. However, a recent set of studies suggests that some sustained attention tasks are mentally taxing (Grier, et al., 2003; Helton, et al., 2005; Helton \& Warm, 2008; Warm, Matthews \& Finomore, 2008). Performance depends on the availability of metabolic and cognitive resources, and as the amount of resources decreases, so does the level of performance. The addition of a secondary task requiring additional resources may result in an accelerated decline in performance. Further work is needed to disambiguate these possibilities.

\section{Conclusion}


Strategic secondary task performance has the effect of increasing alertness as measured by EEG, and improving driving performance, as measured by lane keeping. Performance improvement occurs in conditions of monotony (Atchley \& Chan, 2011) as well as under conditions designed to cause fatigue. Sustained dual-tasking does not lead to the same improvement and also appears to incur costs. What we do not know is if this effect spans the range of conditions that cause losses in alertness. Further work must be conducted to establish if tasks that lead to alertness declines due to resource depletion can also benefit from strategic multi-tasking, or if under these circumstances, another task just makes things worse. 


\section{KEY POINTS}

- $\quad$ An interactive verbal task when fatigued can improve driving performance.

- $\quad$ The verbal task improves driver alertness as measured by EEG.

- Continuously talking eliminates the benefit and incurs performance costs.

- Lateral control measures need further review as metrics of driver alertness. 


\begin{abstract}
Author Information
Paul Atchley is a professor of psychology and director of the Visual Information Processing Laboratory in the Department of Psychology at the University of Kansas. He received his PhD in psychology from the University of California, Riverside, in 1996. Mark Chan is a graduate of the Visual Information Processing Lab at the University of Kansas, where he received his Ph.D. in psychology in 2012. Sabrina Gregersen is a graduate of the University of Kansas, where he received her B.A. in psychology in 2010.
\end{abstract}




\section{Reference}

Atchley, P., Dressel, J., Jones, T. C., Burson, R. A., \& Marshall, D. (2011). Talking and driving: applications of crossmodal action reveal a special role for spatial language. Psychological Research, 75(6), 525-534.

Atchley, P., \& Chan, M. (2011). Potential benefits and costs of concurrent task engagement to maintain vigilance. Human Factors: The Journal of the Human Factors and Ergonomics Society, 53(1), 3-12. doi: 10.1177/0018720810391215

Atchley, P., \& Dressel, J. (2004). Conversation limits the functional field of view. Human Factors, 46(4), 664-673.

Becic, E., Dell, G., Bock, K., Garnsey, S., Kubose, T., \& Kramer, A. (2010). Driving impairs talking. Psychonomic Bulletin \& Review, 17, 15-21.

Berka, C., Levendowski, D. J., Lumicao, M. N.,Yau, A., Davis, G., Zivkovic, V. T., Olmstead, R.E., Tremoulet, P. D., \& Craven, P. L. (2007). EEG correlates of task engagement and mental workload in vigilance, learning, and memory tasks. Aviation, Space and Environmental Medicine, 78, 231-244.

Bradley, M. M., \& Lang, P. J. (1999). Affective norms for English words (ANEW). Gainsville, FL: The NIMH Center for the Study of Emotion and Attention, University of Florida.

Brookhuis, K. A., \& De Waard, D. (1993). The use of psychophysiology to assess driver status. Ergonomics, 36(9), 1099 - 1110.

Brown, I. D. (1994). Driver Fatigue. Human Factors, 36, 298-314.

Cerezuela, G. P., Tejero, P., Choliz, M., Chisvert, M., \& Monteagudo, M. J. (2004). Wertheim's hypothesis on 'highway hypnosis': empirical evidence from a study on motorway and conventional road driving. Accident Analysis \& Prevention, 36(6), 1045-1054. 
Cooper, J. M., Medeiros-Ward, N., \& Strayer, D. L. The impact of eye movements and cognitive workload on lateral position variability in driving. Human Factors, in press.

Deboeck, P. R., Atchley, P., Chan, M., Fries, C., \& Geldhof, J. (2011). Using momentary derivative estimates to gauge driver performance. Advances in Transportation Studies (Special issue).

Desmond, P. A., Hancock, P.A., \& Monette, J.L. (1998). Fatigue and automation-induced impairments in simulated driving performance. Transportation Research Record: Journal of the Transportation Research Board, 1628, 8-14.

Desmond, P. A., \& Matthews, G. (1997). Implications of task-induced fatigue effects for invehicle countermeasures to driver fatigue. Accident Analysis \& Prevention, 29, 515-523.

Driskell, J. E., \& Mullen, B. (2005). The efficacy of naps as a fatigue countermeasure: A metaanalytic integration. Human Factors: The Journal of the Human Factors and Ergonomics Society, 47(2), 360-377. doi: 10.1518/0018720054679498

Drory, A. (1985). Effects of rest and secondary task on simulated truck-driving task performance. Human Factors, 27, 201-207.

Engström, J., Johansson, E., \& Östlund, J. (2005). Effects of visual and cognitive load in real and simulated motorway driving. Transportation Research Part F: Traffic Psychology and Behaviour, 8(2), 97-120.

Foucher, J.R., Otzenberger, H. \& Gounot, D. (2004). Where arousal meets attention: a simultaneous fMRI and EEG recording study. NeuroImage, 22, 688-697.

Gershon, P., Ronen, A., Oron-Gilad, T., \& Shinar, D. (2009). The effects of an interactive cognitive task (ICT) in suppressing fatigue symptoms in driving. Transportation Research Part F, 12, 21-28. 
Gershon, P., Shinar, D., Oron-Gilad, T., Parmet, Y., \& Ronen, A. (2011). Usage and perceived effectiveness of fatigue countermeasures for professional and nonprofessional drivers. Accident Analysis \& Prevention, 43(3), 797-803.

Grier, R. A., Warm, J. S., Dember, W. N., Matthews, G., Galinsky, T. L., Szalma, J. L. \& Parasuraman, R. (2003). The vigilance decrement reflects limitations in effortful attention, not mindlessness. Human Factors, 45, 349-359.

Helton, W.S., Hollander, T.D., Warm, J.S., Matthews, G., Dember, W.N., Wallaart, M., Beauchamp, G., Parasuraman, R. \& Hancock, P.A. (2005). Signal regularity and the mindlessness model of vigilance. British Journal of Psychology, 96, 249-261.

Helton, W.S., \& Warm, J.S. (2008) Signal salience and the mindlessness theory of vigilance. Acta Psychologia, 129, 18-25.

Jasper, H. H. (1958). The ten-twenty electrode system of the International Federation. Electroencephalography and Clinical Neurophysiology, 10(2), 371-375.

Kahneman, D. (1973). Attention and Effort. Englewood Cliffs, NJ: Prentice Hall.

Kunar, M.A., Carter, R., Cohen, M. \& Horowitz, T.S. (2008). Telephone conversation impairs sustained visual attention via a central bottleneck Psychonomic Bulletin \& Review, 15, 1135-1140.

Lal, S. K. L., \& Craig, A. (2002). Driver fatigue: Electroencephalography. Psychophysiology, 39, 313-321.

Liang, Y., \& Lee, J. D. (2010). Combining cognitive and visual distraction: Less than the sum of its parts. Accident Analysis \& Prevention, 42(3), 881-890.

Manly, T., Robertson, I.H., Galloway, M., \& Hawkins, K. (2009). The absent mind: further investigations of sustained attention to response. Neuropsychologia, 37, 661-670. 
Matthews, G., Campbell, S. E., Falconer, S., Joyner, L. A., Huggins, J., Gilliland, K., .. . Warm, J. S. (2002). Fundamental Dimensions of Subjective State in Performance Settings : Task Engagement, Distress, and Worry. Emotion, 2(4), 315-340.

McEvoy, S. P., Stevenson, M. R., McCartt, A. T., Woodward, M., Haworth, C., Palamara, P., \& Cercarelli, R. (2005). Role of mobile phones in motor vehicle crashes resulting in hospital attendance: a case-crossover study. BMJ. doi: 10.1136/bmj.38537.397512.55

Mehler, B., Reimer, B., Coughlin, J., \& Dusek, J. (2009). Impact of Incremental Increases in Cognitive Workload on Physiological Arousal and Performance in Young Adult Drivers. Transportation Research Record: Journal of the Transportation Research Board, 2138, 6-12. doi: 10.3141/2138-02

National Highway Traffic Safety Administration. (2011). Drowsy driving (DOT HS 811 449). Retrieved from http://www-nrd.nhtsa.dot.gov/pubs/811449.pdf

Nguyen, L. T., Jauregui, B., \& Dinges, D. F. (1998). Changing behavior to present drowsy driving and promote traffice safety: Review of proven, promising, and unproven techniques. AAA Foundation for Traffic Safety. Washington, DC. Retrieved from http://www.aaafoundation.org/pdf/drowsydriving.pdf

Nordbakke, S., \& Sagberg, F. (2007). Sleepy at the wheel: Knowledge, symptoms and behaviour among car drivers. Transportation Research Part F: Traffic Psychology and Behaviour, 10, 1-10. doi: 10.1016/j.trf.2006.03.003

Oron-Gilad, T. \& Ronen A. (2007). Road Characteristics and driver fatigue: a simulated study. Traffic Injury Prevention, 8, 1-10.

Oron-Gilad, T., Ronen, A., \& Shinar, D. (2008). Alertness maintaining tasks (AMTs) while driving. Accident Analysis \& Prevention, 40(3), 851-860. doi: 10.1016/j.aap.2007.09.026 
Oron-Gilad, T., \& Shinar, D. (2000). Driver fatigue among military truck drivers. Transportation Research Part F, 3, 195-209.

Paus, T., Zatorre, R. J., Hofle, N., Caramanos, Z., Gotman, J., Petrides, M., \& Evans, A.C. (1997). Time-related changes in neural systems underlying attention and arousal during the performance of an auditory vigilance task. Journal of Cognitive Neuroscience, 9(3), $392-408$.

Pope, A.T., Bogart, E. H., \& Bartolome, D.S. (1995). Biocybernetic system evaluates indices of operator engagement in automated task. Biological Psychology, 40, 187-195.

Prinzel, L. J., Freeman, F. G., Scerbo, M. W., Mikulka, P. J., \& Pope, A. T. (2000). A closedloop system for examining psychophysiological measures for adaptive task allocation. The International Journal of Aviation Psychology, 10(4), 393-410.

Rakauskas, M. E., Gugerty, L. J., \& Ward, N. J. (2004). Effects of naturalistic cell phone conversations on driving performance. Journal of Safety Research, 35, 453-464.

Ranney, T. A., Harbluk, J. L., \& Noy, Y. I. (2005). Effects of Voice Technology on Test Track Driving Performance: Implications for Driver Distraction. Human Factors: The Journal of the Human Factors and Ergonomics Society, 47(2), 439-454. doi: $10.1518 / 0018720054679515$

Redelmeier, D. A., \& Tibshirani, R. J. (1997). Association between cellular-telephone calls and motor vehicle collisions. The New England Journal of Medicine, 336, 453-458.

Recarte, M. A., \& Nunes, L. M. (2000). Effects of verbal and spatial-imagery tasks on eye fixations while driving. Journal of Experimental Psychology: Applied; Journal of Experimental Psychology: Applied, 6(1), 31. 
Reyner, L. A., \& Horne, J. A. (1998). Evaluation of 'in-car' countermeasures to sleepiness: Cold air and radio. Sleep, 21(1), 46-50.

Robertson, I.H., Manly, T., Andrade, J., Baddeley, B.T., \& Yiend, J. (2007). 'Oops!':

Performance correlates of everyday attentional failures in traumatic brain injured and normal subjects. Neuropsychologia, 35, 747-758.

Shomstein, S., \& Yantis, S. (2004). Control of attention shifts between vision and audition in human cortex. The Journal of Neuroscience, 24(47), 10702-10706.

Stoffregen, T. A., Pagulayan, R. J., Bardy, B. G., \& Hettinger, L. J. (2000). Modulating postural control to facilitate visual performance. Human Movement Science, 19, 203-220.

Strayer, D. L., \& Drews, F. A. (2007). Cell phone induced driver distraction. Current Directions in Psychological Science, 16(3), 128-131.

Takayama, L., \& Nass, C. (2008). Assessing the Effectiveness of Interactive Media in Improving Drowsy Driver Safety. Human Factors, 50(5), 772-781.

Thiffault, P., \& Bergeron, J. (2003). Monotony of road environment and driver fatigue: a simulator study. Accident Analysis and Prevention, 35, 381-391.

Verwey, W. B., \& Zaidel, D. M. (1999). Preventing drowsiness accidents by an alertness maintenance device. Accident Analysis \& Prevention, 31(3), 199-211. doi: $10.1016 / \mathrm{S} 0001-4575(98) 00062-1$

Warm, J. S., Matthews, G., \& Finomore, V. S. (2008). Vigilance, Workload, and Stress. In P. A. Hancock, \& Szalma, J.L. (Ed.), Performance Under Stress (pp. 115-141). Burlington: Ashgate Publishing Company.

Warm, J. S., Parasuraman, R., \& Matthews, G. (2008). Vigilance requires hard mental work and is stressful. Human Factors, 50(3), 433-441. 
Wertheim, A. (1978). Explaining highway hypnosis: Experimental evidence for the role of eye movements. Accident Analysis \& Prevention, 10, 111-129.

Table 1

Pre and post task mean (SD) DSSQ engagement scores.

$\underline{\text { Pre-task }} \quad \underline{\text { Post-task }}$

$\underline{\text { Task }}$

No Verbal

$85.00(11.18)$

$45.90(17.52)$

Late Verbal

$76.10(7.21)$

$52.00(9.84)$

Continuous Verbal

$77.00(11.66)$

$50.20(15.01)$

Radio Show

80.00 (9.07)

$57.20(13.23)$ 
Table 2

Mean (SD) proportion of billboards recalled for each verbal task condition.

Task

Billboard Recall (\%)

\begin{tabular}{lc}
\hline No Verbal & $64.3(12.1)$ \\
Late Verbal & $64.3(20.5)$ \\
Continuous Verbal & $34.3(22.5)$ \\
Radio Show & $48.6(18.1)$ \\
\hline
\end{tabular}


Figure 1. SDLP (standard deviation of lane position in meters) across all experimental blocks for the four secondary task conditions (no verbal task, late verbal task, continuous verbal task and radio show).

Figure 2. Engagement index (axis reversed to illustrate decrease in values indicate an increase in engagement) across all experimental blocks for the four secondary task conditions (no verbal task, late verbal task, continuous verbal task and radio show). 